\title{
The pitch and yaw motion coupling research on bionic arm
}

\author{
Hu Tieyu ${ }^{a,{ }^{*}}$, Song Limin ${ }^{b}$, Zhao Kai $^{c}$, Liuxiujuan ${ }^{c}$ and Wang chunguang ${ }^{c}$ \\ Aviation University of Air Force, Changchun, Jilin, China \\ alisong5@126.com, bliminsong_2001@163.com, cwangchungguang@sina.com
}

Keywords: bionic arm; pitch; yaw; motion coupling

\begin{abstract}
The applications of redundant manipulator are more and more widely in the automobile manufacturing industry and aerospace fields. In this paper, the omnidirectional joint unit mechanism is improved and redundant snake-like manipulator of full range PYR series is designed. The composition, working principle, performance index and the joint improved structure of PY (PYR) singular omnidirectional joint are introduced. The formulas of improved PYR type joint also are given. The same as, two types of joint design are combined into a 11 degree of freedom 5 joint robot arm which using slider crank mechanism to solve the pitch and yaw motion coupling problem.
\end{abstract}

\section{Introduction}

The redundant manipulator is increasingly important role in the modernization and national defense. The modular, miniaturization, lightweight, and combined design of mechanical arm are more and more important.Its significance lies in: universality, interchangeability and convenient maintenance, arbitrary combination according to the need, thus it brings the flexibility to use and maintenance convenience. So far, A variety of redundant robot systems have developed on the domestic and abroad, and it is more and more widely used. Some redundant robot systems have been implemented in commercial [1]. The 7-DOF MIA ARM used to study passive impedance control is developed by Japan's Waseda University. The K/B-2017 robot arms are designed by the United States Robotics Research company in 1987, and a hyper redundant robot 32-DOF is developed by the United States aerospace laboratory[2]. The robot dual arm coordination system is developed by the computer department of Nankai University, which has two arms coordination redundant robot system [3].

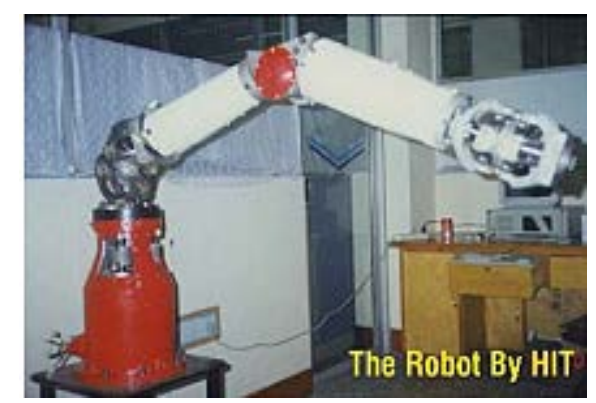

Fig. 1 Apery arm of 7-freedom constituted by Improved PYR all-orientation joint II

\section{The main parameters of improved PY and PYR type all-round joint unit mechanism}

The design of PY type and PYR type omnibearing joint mechanism by using the 3D modeling software CATIA is completed[4]. The motion simulation is conducted in ADAMS software. The pitch (pitch), swing (yaw), (roll) rotation angle value are measured, and the result showed that the structure is reasonable. In order to reduce the weight of joint unit, the seat tube, base were hard aluminum structure and the gear was steel material. A small, lightweight design requirements is reached. The overall size of joints as shown in figure 2. The main parameters of PY joints and PYR joints as shown in Table 1 and Table 2. The pitch and yaw angle value is a single input direction movement angle value, the gear drived, output end angle value is two times the input angle values. 
Table 1 Parameters of PY type joint

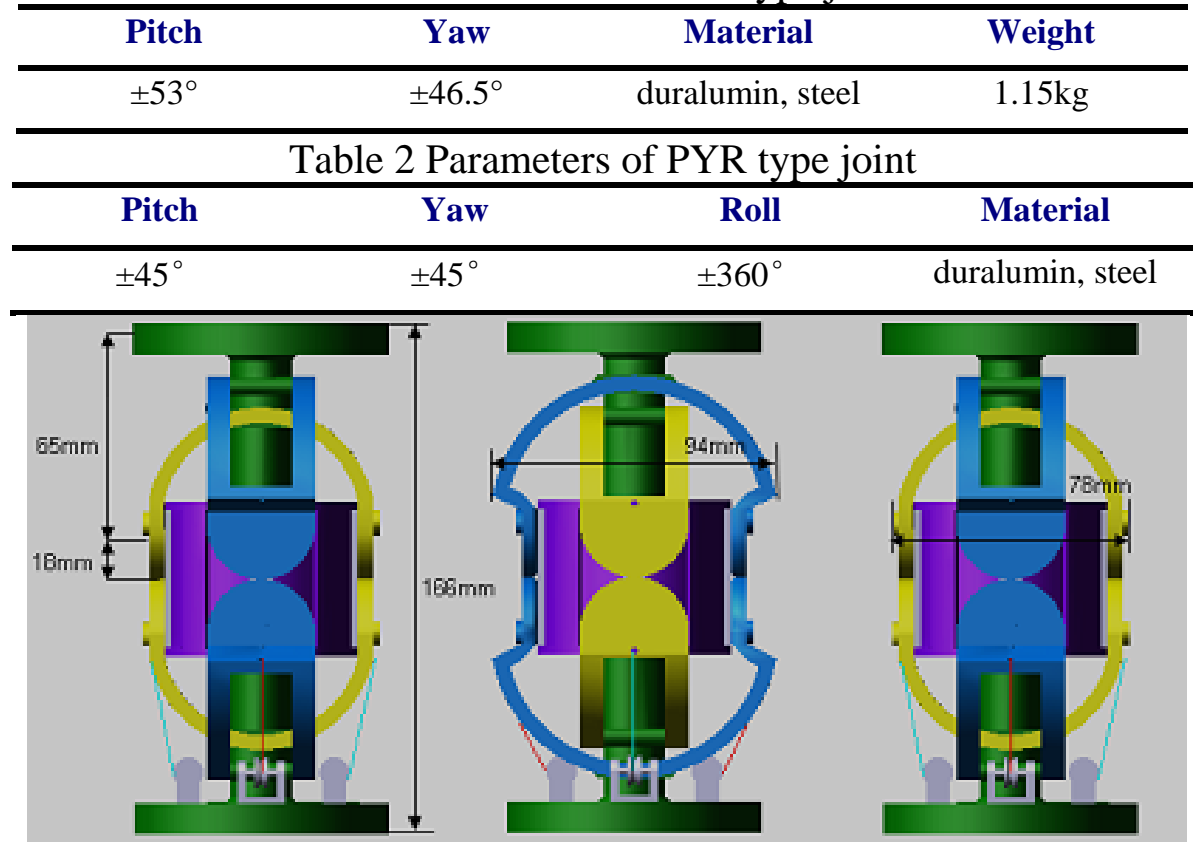

Fig.2 Total dimension of PY joint

\section{The kinematics analysis of improved PYR type joint}

\section{The kinematics of positive solutions of improved PYR joint}

In this paper the improved PYR type joint is studied based on PYR I and PYR II joint. Its mechanism is the same as PYR I and PYR II joint. Therefore, the kinematics analysis of PYR I and PYR II joint is still applicable in this paper[5]. By mechanism analysis the base coordinate system is established as shown in Figure 3.

The positive solutions for terminal attitude is:

$$
\left\{\begin{array}{l}
\alpha^{\prime}=2 \alpha=2 \arccos \frac{|\cos \theta \cos \varphi|}{\sqrt{\cos ^{2} \theta+\sin ^{2} \theta+\cos ^{2} \varphi}} \\
\beta^{\prime}=\beta=\arccos \frac{|\cos \theta \sin \varphi|}{\sqrt{\cos ^{2} \theta \sin ^{2} \varphi+\sin ^{2} \theta \cos ^{2} \varphi}} \\
\psi^{\prime}=\psi
\end{array}\right.
$$

The coordinate value of end points in the basic coordinates is:

$$
\left\{\begin{array}{l}
x_{B}=\left[l_{1} \sin \alpha+l_{2} \sin (2 \alpha)\right] \cos \beta \\
y_{B}=\left[l_{1} \sin \alpha+l_{2} \sin (2 \alpha)\right] \sin \beta \\
z_{B}=l_{1} \cos \alpha+l_{2} \cos (2 \alpha)
\end{array}\right.
$$

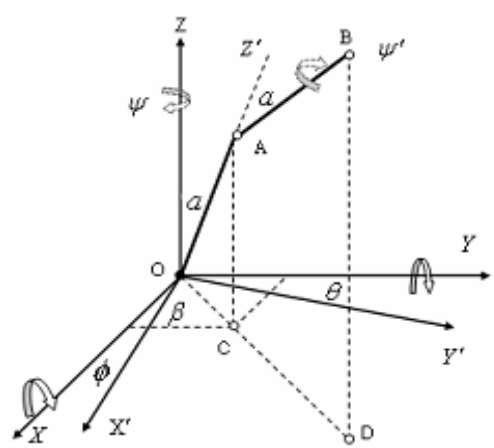

Fig.3 Motion Analysis of PITCH-ROLL-YAW Mechanism 
The inverse solution and velocity Jacobian matrix of improved PYR type joint

The kinematics inverse solution [6] is:

$$
\begin{aligned}
& \left\{\begin{array}{l}
\theta=\kappa_{1} \operatorname{arctg}|\sin \beta \operatorname{tg} \alpha| \\
\varphi=\kappa_{2} \operatorname{arctg}|\cos \beta \operatorname{tg} \alpha| \\
\psi^{\prime}=\psi
\end{array}\right. \\
& {\left[\begin{array}{c}
\dot{\theta} \\
\dot{\varphi} \\
\dot{\psi}
\end{array}\right]=J_{1}\left[\begin{array}{c}
\dot{\alpha} \\
\dot{\beta} \\
\dot{\psi}
\end{array}\right]}
\end{aligned}
$$

Where: $\mathrm{J}_{1}$ is a Jacobian matrix:

$$
\begin{gathered}
J_{1}=\left[\begin{array}{ccc}
\kappa_{1} \frac{\sin \beta \cos ^{2} \theta}{\cos ^{2} \alpha} & \kappa_{1} \cos ^{2} \theta \cos \beta \operatorname{tg} \alpha & 0 \\
\kappa_{2} \frac{\cos \beta \cos ^{2} \varphi}{\cos ^{2} \alpha} & -\kappa_{1} \sin \beta \cos ^{2} \varphi t g \alpha & 0 \\
0 & 0 & 1
\end{array}\right] \\
{\left[\begin{array}{c}
\dot{x}_{B} \\
\dot{y}_{B} \\
\dot{z}_{B}
\end{array}\right]=J_{1}\left[\begin{array}{c}
\dot{\alpha} \\
\dot{\beta} \\
\dot{\psi}^{\prime}
\end{array}\right]}
\end{gathered}
$$

Among them:

$$
\begin{gathered}
J_{2}=\left[\begin{array}{ccc}
{\left[l_{1} \cos \alpha+2 l_{2} \cos 2 \alpha\right] \cos \beta} & {\left[l_{1} \sin \alpha+l_{2} \sin 2 \alpha\right] \sin \beta} & 0 \\
{\left[l_{1} \cos \alpha+2 l_{2} \cos 2 \alpha\right] \sin \beta} & {\left[l_{1} \sin \alpha+l_{2} \sin 2 \alpha\right] \cos \beta} & 0 \\
-l_{1} \sin \alpha+2 l_{2} \sin 2 \alpha & 0 & 1
\end{array}\right] \\
{\left[\begin{array}{c}
\dot{\theta} \\
\dot{\varphi} \\
\dot{\psi}
\end{array}\right]=J_{1} J_{2}^{-1}\left[\begin{array}{c}
\dot{x}_{B} \\
\dot{y}_{B} \\
\dot{z}_{B}
\end{array}\right]}
\end{gathered}
$$

A full range of joint can be simplified as ROLL-PITCH-PITCH-ROL mechanism in figure 4.



Fig.4 ROLL-PITCH-PITCH-ROLL Mechanism 


\section{Design for robot arm joint of 11 freedom degree}

Redundant manipulator has good flexibility and high outer contour adaptability to by surrounded grabbing objects [7]. So it is has very high application value on in the nuclear industry, space station and space object surrounded grabbing operation. Based on the above advantages, the mechanical arm mechanism 5 joints and 11 freedom degrees is design. The joint of the frame and the middle section already use PY two degree freedom joint unit, and the wrist is design into PYR three freedoms. Each joint used cable and guide wheel drive mode, which can arranged on the mechanical arm frame in most cable and a drive motor.The arm length is $1055 \mathrm{~mm}$, the length of joint unit is $166 \mathrm{~mm}$.The 5 joint,11 freedom mechanical arm design of 3-D virtual prototype is shown in figure 5.

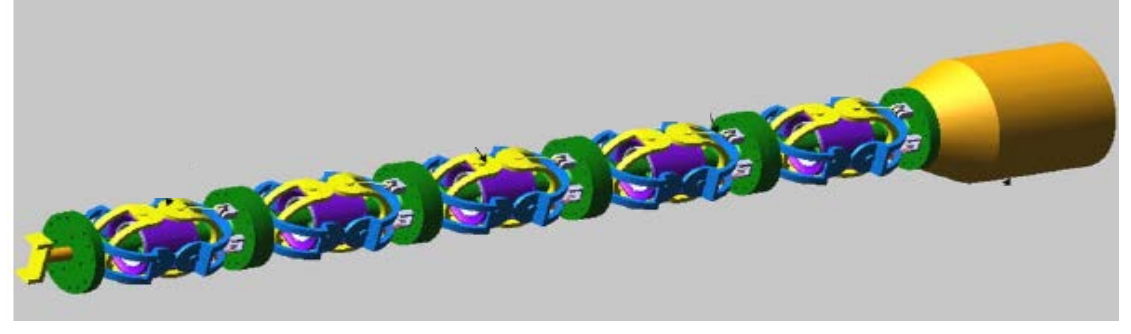

Fig5 Virtual phototyping of the manipulator with 5 joints and 11 freedoms

\section{Conclusions}

Multi redundant manipulator can overcome the shortcome of flexibility, adaptability to environment for the traditional, non redundant robot manipulator. And it can improve the operational flexibility, obstacle avoidance ability. It also has the very good operability and practical value,such as the space technology of space surrounding capture and recovery, complex environment and so on.

The composition, working principle, performance index and joint structure of PY (PYR) singular omnidirectional joint are improved, and the kinematics calculation formula of PYR type joint is given. Two types joint are design to combined into a 11 degree of freedom, 5 joint manipulator, which reached the design requirements for modular, light-weight, miniaturization.

In future, according to the principle of bionics crawling and a full range of joint mechanism design and kinematics theory, the bionic worming crawling motion planning for the 511 degree of freedom manipulator is established.

\section{References}

[1]Ni Shoudong, Yuan Zuqiang, Wen Jufeng. Research actuality of redundant robot mechanism. Journal of Nanjing University of Technology. 2002,24(4):107 110

[2]P K James, M T Jac, V Havard. A Dual-Arm Dexterous Manipulators System with Anthropomorphic Kinematics. Proc.IEEE Int. conf on Robotics and Automation. 1990,368 373

[3] Siciliano B,Kinematic Control of Redundant Robot Manipulators:A Turorial of Intelligent and Robotic system. 1990,3:201 212

[4]Dubey,R and Luh.J.U. S,Redundant Robot Control for Higher flexibilitu, Pro.of IEEE.Int.Conf:Robotics and Automation Raleigh,NC.1987,1066 1072

[5] Liang Feng, Wu Weiguo, Wang Yu, Cai Hegao. A full range of improved PYR joint design, research and application in the flexible arm. Mechanical design and manufacturing. 2006,6:106 108 [6] Wampler C W. Inverse kinematic functions for redundant manipulator. Proc IEEE Int Conf On Rob and Auto. 1987,1212 1218

[7] HollerbachJM. Redundancy resolution of manipulator through torque optimization. Proc IEEE Int Conf On Rob and Auto. 1985,1016 1021 